\title{
Intravenous dexamethasone for the prophylaxis of postoperative nausea and vomiting after neuraxial administration of long-acting opioids: a systematic review and meta-analysis
}

\author{
Grape $\mathrm{S}^{1}$, Albrecht $\mathrm{E}^{2}$ \\ ${ }^{1}$ Hopital du Valais, Dept of Anaesthesiology \& Intensive Care, Sion, Switzerland, email: sina.grape@hopitalvs.ch \\ ${ }^{2}$ Lausanne University Hospital, Dept of Anaesthesiology, Lausanne, Switzerland
}

Background: Neuraxial administration of long-acting opioids provides effective analgesia but is associated with postoperative nausea and vomiting (PONV). Intravenous (IV) dexamethasone has been reported to prevent this side-effect, but with conflicting results*. We performed a systematic review to assess the prophylactic effect of IV dexamethasone on PONV in patients receiving neuraxial long-acting opioids.

Methods: We searched the electronic databases MEDLINE, EMBASE, the Cochrane Central Register of Controlled Trials, the Web of Science and LILACS. We followed the PRISMA statement guidelines. Our primary outcome was the rate of PONV at 24 postoperative hours ( $\mathrm{PO} h$ ), analysed according to the type of comparator: IV dexamethasone versus placebo or IV dexamethasone versus IV dopamine receptor antagonists. Secondary outcomes were pain scores (converted to an analogue scale, 0-10) and need for rescue analgesics, all measured at $24 \mathrm{PO} h$. Meta-analyses were performed with "Review Manager" software (RevMan_version_5.1.6).

Results and discussion: We included 11 randomized controlled trials with a total of 908 patients. In 7 trials opioids were injected intrathecally and in 4 trials epidurally.
Authors used morphine in 9 trials, meperidine in 1 trial and pethidine in 1 trial. At $24 \mathrm{PO} h$ patients receiving IV dexamethasone had a significantly reduced rate of $\mathrm{PONV}$ compared to placebo [risk ratio $=0.4 ; 95 \% \mathrm{Cl}: 0.35 ; 0.64 ; \mathrm{p}<0.00001$; figure 1]. Compared to placebo, the dexamethasone group had a reduction in pain scores at $24 \mathrm{PO} \mathrm{h}$ [mean difference $0.7 ; 95 \% \mathrm{Cl}:-1.2 ;-0.1 ; p=0.02$ ] and in need for rescue analgesics [risk ratio $=0.7 ; 95 \% \mathrm{Cl}$ : $0.6 ; 0.9 ; p=0.003$ ]. Neither PONV, pain scores nor the need for rescue analgesics differed significantly between patients who received dexamethasone or dopamine receptor antagonists.

Figure 1: Rate of PONV at 24 PO hours

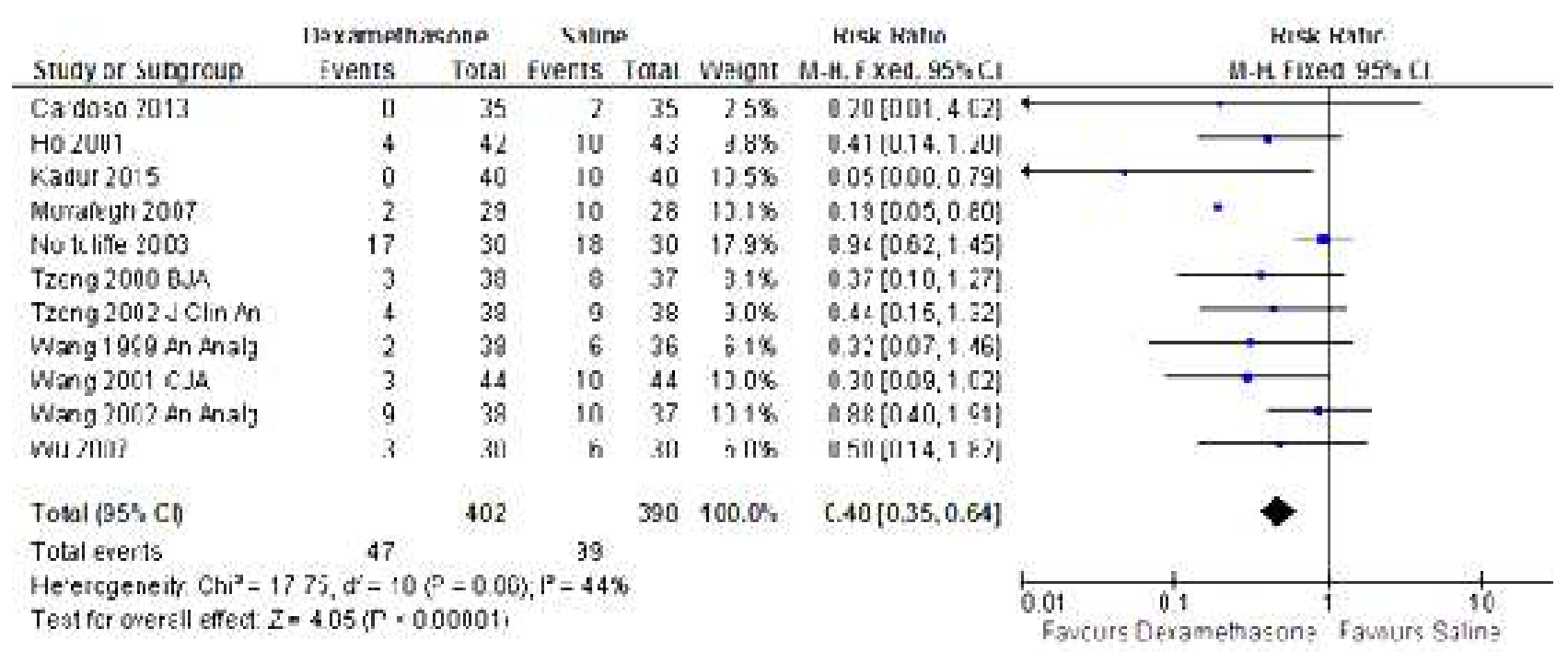

Conclusion: Compared to placebo but not to dopamine receptor antagonists, IV dexamethasone reduces PONV, pain scores and the need for rescue analgesics at $24 \mathrm{PO} \mathrm{h}$ after neuraxial administration of long-acting opioids.

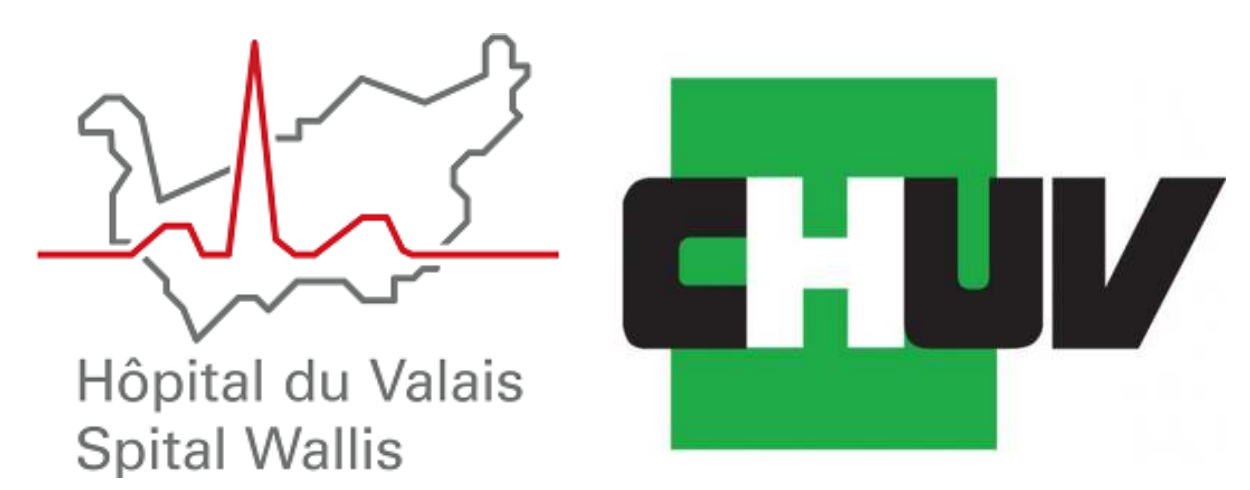

